\title{
Electron Diffraction for Characterization of Nanocrystal Materials: Beyond the Crystal Structure
}

\author{
Z Huang ${ }^{1}, X$ Zou $^{2}$ \\ ${ }^{1}$ Stockholm University, Stockholm, ${ }^{2}$ Stockholm University, Stockholm, Sweden \\ zhehao.huang@mmk.su.se
}

Crystalline solids, such as metal, metal oxides, zeolites, and metal-organic frameworks (MOFs), have been intensively investigated both as traditional and state-of-the-art materials. They have been implemented in a wide range of applications from industrial use to laboratorial research. The applications are closely associated with the physical and chemical properties, which are determined by the atomic crystal structures. Thus, structure determination is arguably the most important characterization step for crystalline materials. Although single crystal X-ray diffraction (SCXRD) is the most practiced and routine method for structure determination, the acquisition of adequate data quality from weakly scattering nano- and micro-sized crystals remains a challenge. While powder Xray diffraction is more suitable technique for handling small crystals, structure determination can be challenging due to severe peak overlap as a consequence of large lattices, complexity of the structures themselves, as well as phase mixtures. Microcrystal electron diffraction (MicroED)[1] or 3-dimensional electron diffraction (3D ED)[2,3] techniques have shown to be powerful for structural determination of 'intractable' crystals that are too small for SCXRD analysis. These techniques benefit from the strong interaction between electrons and matter. Compared to $\mathrm{X}$-ray, electrons generate much higher signal-to-noise ratios, even when the volume of the crystals are 6 or 7 orders of magnitude smaller. A series of ED patterns can be acquired by tilting a crystal around the goniometer axis of a transmission electron microscope (TEM). After reconstruction of the 3D reciprocal lattice, and extracting intensities, the acquired ED data can be applied as SCXRD-like data for structural analysis. We have successfully applied the ED techniques for ab initio structure determination of novel nanocrystalline zeolites and MOFs[4-7], and demonstrated that the technique can achieve an accuracy comparable to SCXRD[8]. Because zeolites and MOFs are sensitive to radiation damage, fast data acquisition is key to the analysis. Nowadays, a complete data can be acquired in 15-150 seconds using continuous goniometer tilt, and the electron dose can be limited to below $0.1 \mathrm{e}$ $\AA$-2. As a consequence, important details beyond the crystal structure can be revealed. For example, the preferred positions of solvents can be determined, and hydrogen bonding can be identified between the solvent molecules and the framework[4]. Such key information of guest-host interactions plays a key role in understanding adsorption properties of porous materials. Moreover, using the MicroED/3D ED method, the positions of different metal cations in MOFs can be directly determined, which is crucial for obtaining key knowledge on the catalytic properties[5]. To further automate the structural analysis, serial rotation electron diffraction method[9] was developed for automated data collection, processing, and structure solution. The large number of data makes it possible for quantitative phase analysis, and for detection of minor phases that may not be detectable by X-ray diffraction. Reference (1) Nannenga, B. L.; Shi, D.; Leslie, A. G. W.; Gonen, T. Nat. Methods 2014, 11 (9), $927-$ 930. (2) Wan, W.; Sun, J.; Su, J.; Hovmöller, S.; Zou, X. J. Appl. Crystallogr. 2013, 46 (6), 1863-1873. (3) Kolb, U.; Gorelik, T.; Otten, M. T. Ultramicroscopy 2008, 108 (8), 763-772. (4) Wang, B.; Rhauderwiek, T.; Inge, A. K.; Xu, H.; Yang, T.; Huang, Z.; Stock, N.; Zou, X. Chem. - Eur. J. 2018, 24 (66), 17429-17433. (5) Yuan, S.; Qin, J.S.; Xu, H.-Q.; Su, J.; Rossi, D.; Chen, Y.; Zhang, L.; Lollar, C.; Wang, Q.; Jiang, H.-L.; Son, D. H.; Xu, H.; Huang, Z.; Zou, X.; Zhou, H.-C. ACS Cent. Sci. 2018, 4 (1), 105-111. (6) Roy, S.; Huang, Z.; Bhunia, A.; Castner, A.; Gupta, A. K.; Zou, X.; Ott, S. J. Am. Chem. Soc. 2019, 141 (40), 15942-15950. (7) Carraro, F.; VelásquezHernández, M. de J.; Astria, E.; Liang, W.; Twight, L.; Parise, C.; Ge, M.; Huang, Z.; Ricco, R.; Zou, X.; Villanova, L.; Kappe, C. O.; Doonan, C.; Falcaro, P. Chem. Sci. 2020, 11, 3397-3404. (8) Huang, Z.; Ge, M.; Carraro, F.; Doonan, C. J.; Falcaro, P.; Zou, X. Faraday Discuss. 2020, DOI: 10.1039/D0FD00015A. (9) Wang, B.; Zou, X.; Smeets, S. IUCrJ 2019, 6 (5), 854-867.

Acta Cryst. (2020). A76, a30 